\title{
Production of Antihydrogen
}

G. Baur ${ }^{\text {a }}$, G. Boero ${ }^{\mathrm{b}}$, S. Brauksiepe ${ }^{\mathrm{a}}, \mathrm{A}$. Buzzo ${ }^{\mathrm{b}}$, W. Eyrich ${ }^{\text {, }}$, R. Geyer ${ }^{\text {a }}$, D. Grzonka ${ }^{\text {a }}$, J. Hauffe ${ }^{c}$, K. Kilian ${ }^{\text {a }}$, M. LoVetere ${ }^{\mathrm{b}}$, M. Macri ${ }^{b}$, M. Moosburger ${ }^{c}$, R. Nellen ${ }^{a}$, W. Oelert ${ }^{a}$, S. Passaggio ${ }^{b}$, A. Pozzo ${ }^{b}$, K. Röhrich ${ }^{\text {a }}$, K. Sachs ${ }^{\text {a }}$, G. Schepers ${ }^{e}$, T. Sefzick ${ }^{a}$, R.S. Simon ${ }^{d}$, R. Stratmann ${ }^{\text {, }}$, F. Stinzing ${ }^{c}$ and M. Wolke ${ }^{a}$

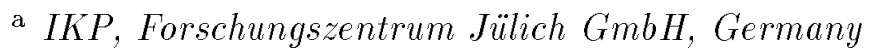

${ }^{\mathrm{b}}$ Genoa University and INFN, Italy

c PI, Universität Erlangen-Nürnberg, Germany

d GSI Darmstadt, Germany

e IKP, Universität Münster, Germany

Results are presented for a measurement for the production of the antihydrogen atom $\overline{\mathrm{H}}^{0} \equiv \overline{\mathrm{p}} \mathrm{e}^{+}$, the simplest atomic bound state of antimatter. A method has been used by the PS210 collaboration at LEAR which assumes that the production of $\overline{\mathrm{H}}^{0}$ is predominantly mediated by the $\mathrm{e}^{+} \mathrm{e}^{-}-$ pair creation via the two-photon mechanism in the antiproton-nucleus interaction. Neutral $\overline{\mathrm{H}}^{0}$ atoms are identified by a unique sequence of characteristics. In principle $\overline{\mathrm{H}}^{0}$ is well suited for investigations of fundamental CPT violation studies under different forces, however, in our investigations we concentrate on the production of this antimatter object, since so far it has never been observed before.

The production of 11 antihydrogen atoms is reported including possibly $2 \pm 1$ background signals, the observed yield agrees with theoretical predictions.

PACS: $25.43 .+\mathrm{t}$

Keyword: Antihydrogen

\section{Introduction}

The idea of the existence of antimatter goes back to the work of Dirac. In 1931 he proposed the positron [1]. Soon afterwards it was confirmed by the detection of electron-positron pairs created in cosmic rays [2]. For each particle 
(meson, baryon, lepton, gauge boson,...) a corresponding antiparticle exists as predicted by the CPT theorem.

The CPT theorem can be derived from very general principles of relativistic quantum field theory. The combined operation of charge conjugation (C), space reflection $(\mathrm{P})$ and time reversal $(\mathrm{T})$ represents an exact symmetry of nature. A determination of CPT invariance is therefore a test of the correctness of the description of the microscopic phenomena in terms of the existing local field theory. CPT violation would mean an existence of unknown properties of the fields and their interactions which are outside the standard theory. The search for effects of CPT violation in different processes is therefore desirable.

It follows from the CPT theorem that particle and antiparticle have the same mass, spin and total life time and the same value but opposite sign of charge and magnetic moment. Since there is no reasonable doubt about the existence of the symmetry between particle and antiparticle, it should be possible to combine antiparticles into antimatter under the same forces as particles bind to form normal matter, in particular, to produce the simplest antiatom out of an antiproton and a positron: $\overline{\mathbf{H}}^{0}$.

Though there is hardly any doubt that $\overline{\mathrm{H}}^{0}$ exists, it has never been observed. Once it is produced $\overline{\mathrm{H}}^{0}$ is certainly an ideal laboratory for studying CPT invariance by comparing interactions in matter and antimatter environments. A special challenge is the possibility of studying hydrogen and antihydrogen under essentially identical or symmetrical experimental conditions.

The ingredients for producing antihydrogen in a state bound electromagnetically are obvious; the two antiparticles $\bar{p}$ and $\mathrm{e}^{+}$must be brought close enough together in energy and momentum space in order to react and combine to form $\overline{\mathrm{H}}^{0}$.

Two principal solutions for $\overline{\mathrm{H}}^{0}$ production, at rest and in flight, have been suggested and are discussed in References [3]-[8]. The variant of the flight method used here has first been suggested by Munger et al. [6]. An antiproton passing through the Coulomb field of a nucleus with charge $\mathrm{Z}$ will create an $\mathrm{e}^{+} \mathrm{e}^{-}$-pair. Occasionally the antiproton will capture a positron from the produced pair and form a fast moving antihydrogen. In lowest order two kinds of mechanisms have been estimated [5],[7]:

(i) the space like $\gamma \gamma$-production of $\mathrm{e}^{+} \mathrm{e}^{-}$-pairs, see Fig.1:

$$
\overline{\mathrm{p}} \mathrm{Z} \rightarrow \overline{\mathrm{p}} \gamma \gamma \mathrm{Z} \rightarrow \overline{\mathrm{p}} \mathrm{e}^{+} \mathrm{e}^{-} \mathrm{Z} \rightarrow \overline{\mathrm{H}}^{0} \mathrm{e}^{-} \mathrm{Z}
$$

(ii) the $\mathrm{e}^{+} \mathrm{e}^{-}$-pair production converted from virtual time like Bremsstrah- 
lung photons:

$$
\overline{\mathrm{p}} \mathrm{Z} \rightarrow \overline{\mathrm{p}} \gamma^{*} \mathrm{Z} \rightarrow \overline{\mathrm{p}} \mathrm{e}^{+} \mathrm{e}^{-} \mathrm{Z} \rightarrow \overline{\mathrm{H}}^{0} \mathrm{e}^{-} \mathrm{Z}
$$

The second mechanism is calculated [8] to be two to three orders of magnitude lower than the $\gamma \gamma$ induced one, which has been evaluated by several authors $[6],[8]$ resulting in a $Z^{2}$ and a $\ln \left(\mathrm{E}_{\overline{\mathrm{p}}} / \mathrm{m}_{\overline{\mathrm{p}}}\right)$ dependence, where $\left(\mathrm{E}_{\overline{\mathrm{p}}} / \mathrm{m}_{\overline{\mathrm{p}}}\right)$ denotes the Lorentz factor. The cross section is expected to be in the order of $2 \mathrm{pb}$ for a $\bar{p}$-momentum around $2 \mathrm{GeV} / \mathrm{c}$, the maximum momentum at LEAR.

Another variant of the $\gamma \gamma$-production was recently studied [9] by an alternative mechanism for pair production in relativistic collisions out of the charge transfer from the negative energy continuum. However, at the present Lorentz factor this mechanism has a negligible cross section.

\section{Experimental Method}

The production of $\overline{\mathrm{H}}^{0}$ requires a rather thin target since otherwise the antiatom would break up into its constituents. However, due to the small production cross section a large luminosity is demanded and therefore only the technique of using a cluster target at an internal antiproton beam seems possible. During JETSET (PS202) experiments at LEAR in 1993 and 1994 experimental conditions for measuring the $\overline{\mathrm{H}}^{0}$ production have been tested parasitically with a hydrogen cluster target and with beam momenta settings according to the JETSET physics program. These investigations are reported elsewhere [10] and resulted in the observation of the characteristic signature of one event, which could not be classified as a significant proof of the existence of $\overline{\mathrm{H}}^{0}$.

The present results were taken in a dedicated beam time period with effectively 15 hours beam on target, distributed over several days of running in parallel to the Crystal Barrel (PS197) experiment. A Xe-cluster target was used [11]. The beam target interaction intensity was optimized such that a fairly constant luminosity was maintained, i.e. the target density was low (about $1 * 10^{12}$ atoms $/ \mathrm{cm}^{2}$ ) at the beginning and was increased to about $3 * 10^{13}$ atoms $/ \mathrm{cm}^{2}$ according to the decrease of the $1.94 \mathrm{GeV} / \mathrm{c} \overline{\mathrm{p}}$-beam intensity. Starting typically with $1.7 * 10^{10} \overline{\mathrm{p}}$ 's in LEAR beam lifetimes of at least 3 minutes were possible with the optimum settings of the LEAR accelerator and cooling system. A typical run lasted for twelve minutes before a new filling of LEAR was done. The relative luminosity was monitored on-line with a three layer circular scintillator hodoscope [12]. Results of this determination will be published later. Here we estimate the integrated luminosity from the number of antiprotons and target thickness to be $\mathcal{L}=5 * 10^{33} \mathrm{~cm}^{-2} \pm 50 \%$ for the total running period in September/October 1995. 
$\overline{\mathrm{H}}^{0}$ is formed in the cluster target at the center of the straight section of LEAR with a preference for $0^{0}$ forward production. The reaction kinematics which leads to a bound $\overline{\mathrm{p}} \mathrm{e}^{+}$-atomic state is very close to the forward part of the $\bar{p} p$ elastic scattering. The maximum transverse momentum is in the order of $0.5 \mathrm{MeV} / \mathrm{c}$ compared to the longitudinal momentum of $1.94 \mathrm{GeV} / \mathrm{c} . \mathrm{A} \overline{\mathrm{H}}^{0}$ production is only possible for $\mathrm{e}^{+}$with a relative energy at or below the hydrogen Rydberg constant of $13.6 \mathrm{eV}$ with respect to $\overline{\mathrm{p}}$. Thus the $\mathrm{e}^{+}$-velocity has to be very close to the $\overline{\mathrm{p}}$-velocity.

Since the cross section of the $\overline{\mathrm{H}}^{0}$ production is expected to be $2 \mathrm{pb} * \mathrm{Z}^{2} \simeq$ $6 * 10^{-33} \mathrm{~cm}^{2}$ a total production rate of $30 \overline{\mathrm{H}}^{0}$ would be expected. According to both test measurements and Monte Carlo calculations the acceptance of the detection system was determined to be $\epsilon=0.3$ which would result in the observation of nine $\overline{\mathrm{H}}^{0}$ with a clear signature during the running period. This number is an upper limit since further acceptance losses are expected due to both the wire chamber efficiency and material interactions in the flight path of the $\overline{\mathrm{p}}$ 's. These effects are estimated to diminish the total detection efficiency by about $15 \%$.

A clear signature for a good event has to fulfill the following criteria (see Fig.2a and Fig.2b for the detector set-up):

i) As a neutral object an $\overline{\mathrm{H}}^{0}$ - produced ten meters upstream in the center of the straight section of LEAR - will exit the accelerator ring tangentially and will be stripped in the first silicon counter still in the LEAR vacuum system. Here both constituents of $\overline{\mathrm{H}}^{0}$ have the same velocity and therefore at a $\bar{p}$ beam momentum of $1.94 \mathrm{GeV} / \mathrm{c}$ the kinetic energy is $0.663 \mathrm{MeV}$ for $\mathrm{e}^{+}$and $1217 \mathrm{MeV}$ for $\overline{\mathrm{p}}$, respectively. These two particles hit simultaneously the first silicon counter $\left(700 \mu \mathrm{m}\right.$ thick and $1200 \mathrm{~mm}^{2}$ active area [13]) which, together with the second one $(500 \mu \mathrm{m}$ thickness and $1200 \mathrm{~mm}^{2}$ ) measures the $\mathrm{dE} / \mathrm{dx}$ of the $\overline{\mathrm{p}}$ plus the kinetic energy of the $\mathrm{e}^{+}$being stopped in these two detectors. The third silicon counter with dimensions the same as the first one should give a signal proportional to $\mathrm{dE} / \mathrm{dx}$ of the $\overline{\mathrm{p}}$ only.

ii) The two $511 \mathrm{keV}$ photons from the $\mathrm{e}^{+}$-annihilation will be detected back to back in the cylindrical six-fold segmented NaI counter [14], which forms an inner and outer diameter of $8 \mathrm{~cm}$ and $40 \mathrm{~cm}$, respectively, covers a solid angle of $91 \%$, has an energy resolution of $14 \%$ FWHM, and provides a $511 \mathrm{keV} \gamma$-detection efficiency of $82 \%$ which has to be multiplied by 0.71 for the peak-to-total ratio [14]. The silicon counter telescope is located in the center of the $\mathrm{NaI}$ crystal arrangement. The $\mathrm{NaI}$ detector was temperature controlled during the runs to $17 \pm 0.5^{\circ} \mathrm{C}$.

iii) The $\overline{\mathrm{p}}$ 's resulting from the stripped $\overline{\mathrm{H}}^{0}$ will continue with a velocity of $\beta=0.900$. They penetrate through a set of three start scintillators, each $4 \mathrm{~mm}$ thick, a scintillator hodoscope of 16 fibers $2 * 2 * 32 \mathrm{~mm}^{3}$ in 
vertical and horizontal orientation, and a group of four stop scintillators arranged as indicated in the figure. All scintillator ADC and TDC values have to be in specific intervals which were determined by cosmic ray calibrations before and after the installation of the detector system in the experimental set-up at LEAR.

iv) The deflection of the registered charged particles was measured with a spectrometer consisting of three horizontally sensitive delay-line readout drift chambers [15] with a used active area of $8 * 8 \mathrm{~cm}^{2}$ and a dipole field varied between $0.05 \mathrm{Tm}$ and $0.15 \mathrm{Tm}$. The deflection should be within the predicted values for the $\overline{\mathrm{p}}$ from $\overline{\mathrm{H}}^{0}$, taking the straggling of $\pm 12 \mathrm{~mm}$ into account due to matter in the flight path.

Since in the present situation no additional well known reaction could be measured in parallel to the reaction to be studied, the entire detector system had to be calibrated before the start of the experiment. These calibrations were done with cosmic rays for the time-of-flight and energy losses, with a ${ }^{22} \mathrm{Na}$ source for the $\mathrm{NaI}$ crystals and with both ${ }^{106} \mathrm{Ru}$ and ${ }^{207} \mathrm{Bi}$ sources for the energy and timing determination for the silicon detectors and the scintillator hodoscope.

\section{Analysis}

The chain of analysis used for the identification of the produced $\overline{\mathrm{H}}^{0}$ employed neither cuts on the time-of-flight nor on the deflection in the magnetic separator. In Fig. 3 the time-of-flight via the signal amplitude from one of the time-of-flight start scintillators is shown. In the range from $17 \mathrm{~ns}$ to $19 \mathrm{~ns}$ the signal amplitude is due to single pion (amplitude $\simeq 130$ ), double and multi pion production. The main source of these mesons is most likely the reaction chain: $\overline{\mathrm{p}} \mathrm{Xe} \rightarrow \overline{\mathrm{n}}$ "anything", where the $\overline{\mathrm{n}}$ annihilates in the silicon detector telescope and produces multi-pion events. The band starting at amplitude values of about 160 and ranging from $19.5 \mathrm{~ns}$ to $30 \mathrm{~ns}$ with decreasing intensity is due to fast and slow protons or antiprotons which probably again are due to secondary reactions from $\bar{n}$ 's leaving the LEAR accelerator tangentially. Here we can not distinguish in general whether the slower baryons are due to additional pion production and/or to any kind of excitation of the Xe target nucleus.

Whereas in Fig.3 the sum of all data taken in 1995 with about 300,000 triggers is shown, Fig.4 presents only a single run with a magnetic field setting of $0.136 \mathrm{Tm}$ which results in an expected deflection of the $\overline{\mathrm{p}}$ 's from $\overline{\mathrm{H}}^{0}$ of $39.5 \mathrm{~mm} \pm 12 \mathrm{~mm}$ and a time-of-flight of $19.7 \pm 0.6 \mathrm{~ns}$ where the allowed range corresponds to $2.5 \sigma$ around the expected value. In Fig.5 again the integral of all data taken at different magnetic field settings is shown now with 
the deflection normalized such that the expected position for the $\overline{\mathrm{p}}$ 's from $\overline{\mathrm{H}}^{0}$ should be zero. Naturally the upper display in Fig.5 includes and selects only those triggers $(\simeq 23,300)$ where a track through all three wire chambers could be defined, but has no additional cut on the data.

Additional cuts were introduced in order to produce the second part of the figure, with 94 events remaining, where now the essential conditions are that:

i) the sum of the signal from the first two silicon detectors is equivalent to the kinetic energy of the stopped $\mathrm{e}^{+}$plus the energy loss of the $\bar{p}$ whereas the third silicon detector gives an energy signal relevant for the $\bar{p}$ energy loss only.

ii) the determined particle trajectory points back into the area of the target region.

iii) the signal amplitude from the time of flight scintillators is in reasonable agreement with the energy loss of the $\overline{\mathrm{p}}$ 's from $\overline{\mathrm{H}}^{0}$.

With these requirements the pionic events disappear as can be seen from the middle part of Fig.5. Essentially only events due to antiprotons are left.

In Figure 6 two-dimensional scatter plots of the energy deposit are shown for a) the three combinations of two opposite $\mathrm{NaI}$ blocks, b) the combinations of non-adjacent but non-opposite NaI blocks, and finally c) the combination of neighboring NaI blocks. Input for this figure is the reduced data set as given in the center of Fig.5, which still includes antiprotons of different velocities. By dotted lines the energy limits for $511 \mathrm{keV} \gamma$ 's are shown which have been determined before and after the actual run using a ${ }^{22} \mathrm{Na}$ source. Figure 6 a shows a clearly separated group of eight $\gamma \gamma$ coincidences with both $\gamma^{\prime}$ s in the $511 \mathrm{keV}$ range. Higher energy deposits in two opposite NaI crystals do not occur. Further, two energy bands are obvious where one NaI crystal has a continuous energy spectrum and the other is significantly below the $511 \mathrm{keV}$ region. Figure 6b shows a similar picture as Fig.6a but only three events indicate a $511 \mathrm{keV}$ $\gamma \gamma$ coincidence. The justification for including the non-adjacent NaI block coincidence condition results from the expectation that not all $\mathrm{e}^{+}$annihilations happen in the center of the silicon counter. Geometrical estimates and Monte Carlo simulations including a $5 \mathrm{~mm}$ displacement of the interaction point between beam and target, an extended target shape of $1 \mathrm{~cm}^{2}$, and the maximal transversal momentum in the antihydrogen production resulted in about $41 \%$ expectation of the non-adjacent but non-opposite NaI coincidence configuration. Finally, in Fig.6c a single event is observed which obeys the $2 * 511 \mathrm{keV}$ condition, but in two neighboring crystals. Such condition might occur from a single Compton scattered high energy $\gamma$, or could be attributed to an accidental $\gamma \gamma$ coincidence. Therefore this one event gives an upper limit of the possible background. We can conclude that Fig.6 demonstrates eleven events which follow the clear signature of antihydrogen being produced, but that the 
possibility of one background event is obvious. All these twelve events show the expected deflection well within $12 \mathrm{~mm}$ and they are in the correct time of flight range of $19.7 \pm 0.6 \mathrm{~ns}$.

\section{Discussion}

A careful investigation of possible background events revealing the signature of an $\overline{\mathrm{H}}^{0}$ production has been done with the collected data. The first background consideration is demonstrated by Fig.6 above. In addition, the signal amplitudes for the first and the second silicon counters are shown in the scatter plots of Fig.7a and Fig.7b for the sum of all identified $\overline{\mathrm{p}}$ 's and for the $\overline{\mathrm{H}}^{0}$ candidates, respectively. Figure $7 \mathrm{~b}$ indicates clearly the large amplitude in the first silicon detector, due to the additional kinetic energy deposit of the $\mathrm{e}^{+}$ stripped from the $\overline{\mathrm{H}}^{0}$. As expected the $0.663 \mathrm{MeV}$ positrons are essentially stopped in the first silicon detector and deposit most of their energy here. A comparison of the two displays in Fig.7 demonstrates that the events in Fig.7b are consistent with $\overline{\mathrm{H}}^{0}$ production and inconsistent with background.

Further, a scatter plot of the $\gamma \gamma$ coincidence for two opposite NaI crystals summed over the three possibilities is shown in Fig. 8 with the condition that no signal is produced in the silicon detectors \#1 and \#2 but any kind of signal in silicon detector \#3. This way it is demonstrated what kind of $\overline{\mathrm{H}}^{0}$ signature could be feigned by the interaction of a neutral particle with the silicon detector material. An effect like that should happen in one of the first two silicon counters with a probability proportional to the relative detector thicknesses $(12 / 7)$. In Fig. 8 one background event shows up resulting in a background estimate of two events being possible in the observed $\overline{\mathrm{H}}^{0}$ sample.

Finally, the off-line trigger condition on the silicon detectors have been exchanged: i.e. i) requiring a signal as large as that due to the sum of both the kinetic $\mathrm{e}^{+}$energy and the $\overline{\mathrm{p}}$ energy loss only in the third detector and ii) signal amplitudes equal or smaller than the energy loss of an $\bar{p}$ only, including even no hit indication in the first two detectors (allowing for a neutral particle). No event was observed. For this background check the cuts on the other detectors of the experiment have been kept unchanged. As above, since the probability for producing an event with an $\overline{\mathrm{H}}^{0}$ signature (where the $\overline{\mathrm{p}}$ must have been produced out of an $\bar{n}$ ) in the $\bar{p}$ interaction with the detector material is the same in the first and in the third silicon detector: such processes can be excluded. The same argument holds for the decay $\overline{\mathrm{n}} \rightarrow \overline{\mathrm{p}} \mathrm{e}^{+} \nu$ which could feign the production of $\overline{\mathrm{H}}^{0}$ but which is already very unlikely to happen in the acceptance of the trigger in use. 
Figure 9 displays the time of flight projection from Fig.3, with the time of flight measured for the $\overline{\mathrm{p}}$ 's due to $\overline{\mathrm{H}}^{0}$ given in the lower part of the figure. As expected, these timing measurements are at the fast end of the antiproton band since the maximum transverse momentum of only $0.5 \mathrm{MeV} / \mathrm{c}$ is negligible compared to the $1.94 \mathrm{GeV} / \mathrm{c}$ beam momentum.

Thus, it can be concluded that $\mathbf{1 1} \overline{\mathbf{H}}^{0}$ atoms have been observed. The $\overline{\mathrm{H}}^{0}$ production rate is in good agreement with the expected value following the theoretical $\gamma \gamma$-production mechanism [6],[8]. No production of $\overline{\mathrm{H}}^{0}$ has ever been reported or observed before.

Based on Poisson statistics the number of background events would be less than three with a confidence level of $95 \%$. This is in agreement with a background estimate of $2 \pm 1$ out of the 11 events.

In the future more $\overline{\mathrm{H}}^{0}$ atoms should be produced and especially under conditions such, that spectroscopy could be done to test the CPT symmetry of matter and anti-matter.

\section{$5 \quad$ Acknowledgements}

We appreciate the help of many people. Especially we would like to thank the PS and the LEAR operation teams for delivering excellent $\bar{p}$ beams, the LEAR vacuum group for making it possible to us to use the Xe cluster target in the ultra high vacuum of LEAR, and the transport and handling group for moving the shielding wall of LEAR.

This work could not have been done without the enormous engagement and continuous support from M. Chanel, C. Lacroix, P. Lefèvre, D. Möhl, and G. Molinari. We like to thank them as well as W. Niebur for technical support with the set-up, T. Sagefka for preparing and calibrating the magnetic system, and J. Spanggaard for helping us with the wire chambers.

We would like to thank R. Landua and E. Vogt for careful reading of the manuscript and for their valuable suggestions. 


\section{References}

[1] P.A.M. Dirac, Proc. Roy. Soc. A117 (1928) 610,

P.A.M. Dirac, Proc. Roy. Soc. A133 (1931) 60

[2] C.D. Anderson, Science 76 (1932) 238

C.D. Anderson, Phys. Rev. 43 (1933) 491

[3] G. Gabrielse et al., Phys. Rev. Lett. 65 (1990) 1317

G. Gabrielse et al., Proceedings of the LEAP'94 conference, Bled, Slovenia, September 12-17, 1994

G. Gabrielse et al., PS196 experiment at LEAR

[4] A. Wolf, AntiProton '86, VIII European Symposium on Nucleon-Antinucleon Interaction, Thessaloniki, Greece

[5] H. Poth et al., Hyperfine Interactions 44 (1988) 259

G. Budker and A.N. Skrinsky, Sov. Phys. Usp. 21 (1978) 227

H. Herr et al., Proceedings of the second Workshop Physics at LEAR, Erice (1982) 659

[6] C.T. Munger et al., Hyperfine Interactions 76 (1993) 175

C.T. Munger et al., Phys. Rev. D 49 (1994) 3228

[7] M. Charlton et al., CERN-PPE/94-18, February 1994

M. Charlton et al., Physics Reports 241 (1994) 65

[8] G. Baur, Phys. Lett. B 311 (1993) 343

[9] J. Eichler, Phys. Rev. Lett. 75 (1995) 3653

[10] G. Baur et al., Proceedings of the International Conference 'Physics with GeVParticle Beams', p.543, Jülich, August 22-25, 1994, Edts.: H. Machner, K. Sistemich, World Scientific

G. Baur et al., Proceedings of the Third Biennial Conference 'Low Energy Antiproton Physics', p.579, Bled, September 12-17, 1994, Edts.: G. Kernel, P. Krizan, M. Mikuz, World Scientific

G. Baur et al., Proceedings of the NAN' 95 Conference 'Nucleon-Anti-Nucleon', Moskau, September 11-16, 1995, to be published in World Scientific

[11] G. Boero, PS210 note number 1, April 10, 1995

[12] M. Dahmen et al., Nucl. Inst. \& Meth. A348 (1994) 97

[13] CANBERRA, Fully depleted PIPS Detectors

[14] H. Keller et al., Nucl. Inst. \& Meth. A300 (1991) 67

[15] A. Manarin and G. Vismara, 'The delay wire chamber (DWC) description', LEP/BI-TA/Note 85-3, CERN Prevessin 
Figures

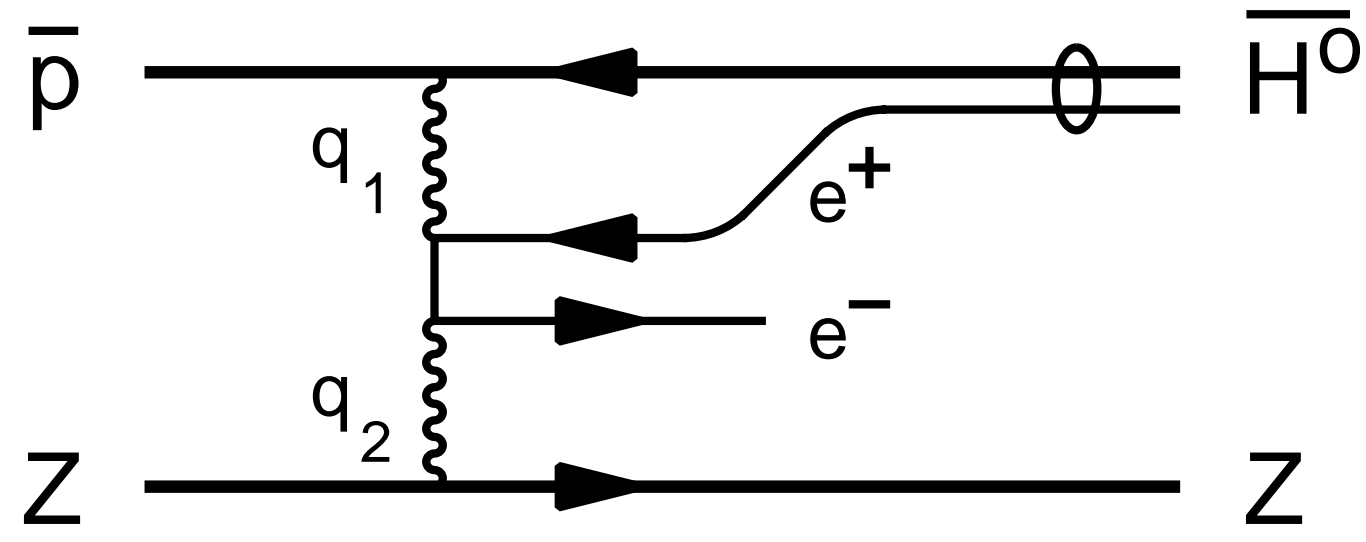

Figure 1: A schematic view of the two-photon mechanism for $\mathrm{e}^{+} \mathrm{e}^{-}$and $\overline{\mathrm{H}}^{0}$ production
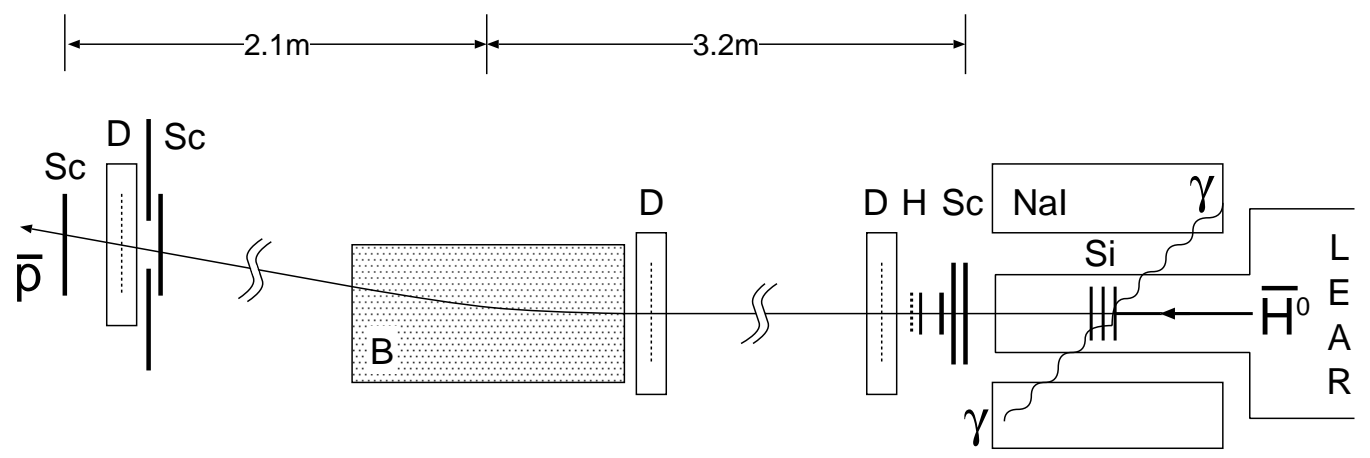

Figure 2: a) Lay-out of the experiment PS210

Sc: trigger and time of flight scintillators

Si: silicon counters

D: Delay wire chambers

NaI: six-fold segmented $\mathrm{NaI}$ detector

$\mathrm{H}$ : scintillating fibre hodoscope

B: magnetic dipole field 


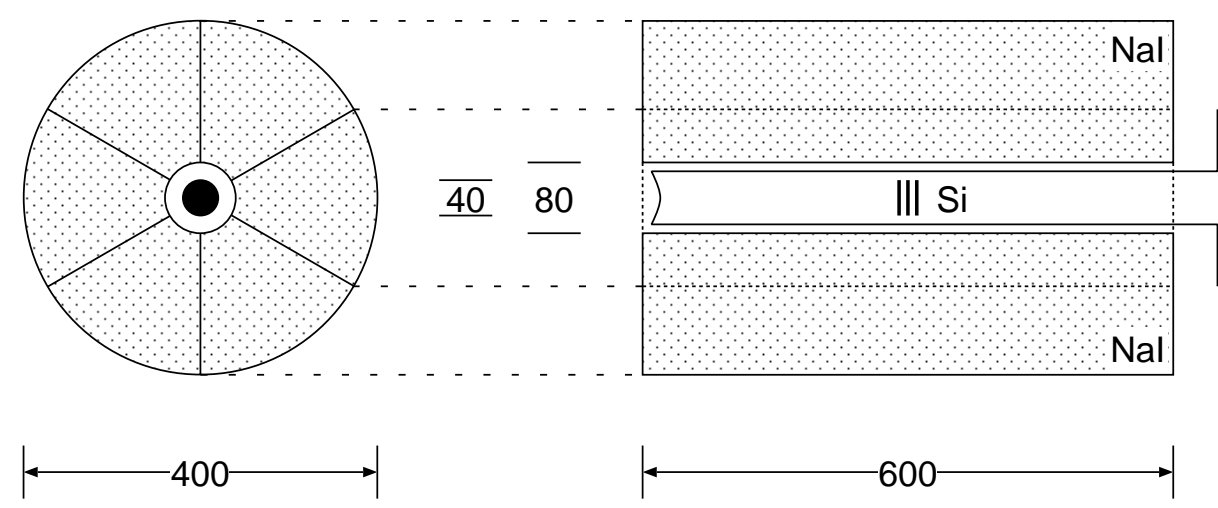

Figure 2: b) A closer look to the NaI/Silicon detector arrangement $\mathrm{Si}$ : three silicon detectors

NaI: six-fold segmented NaI detector dimensions are in $\mathrm{mm}$

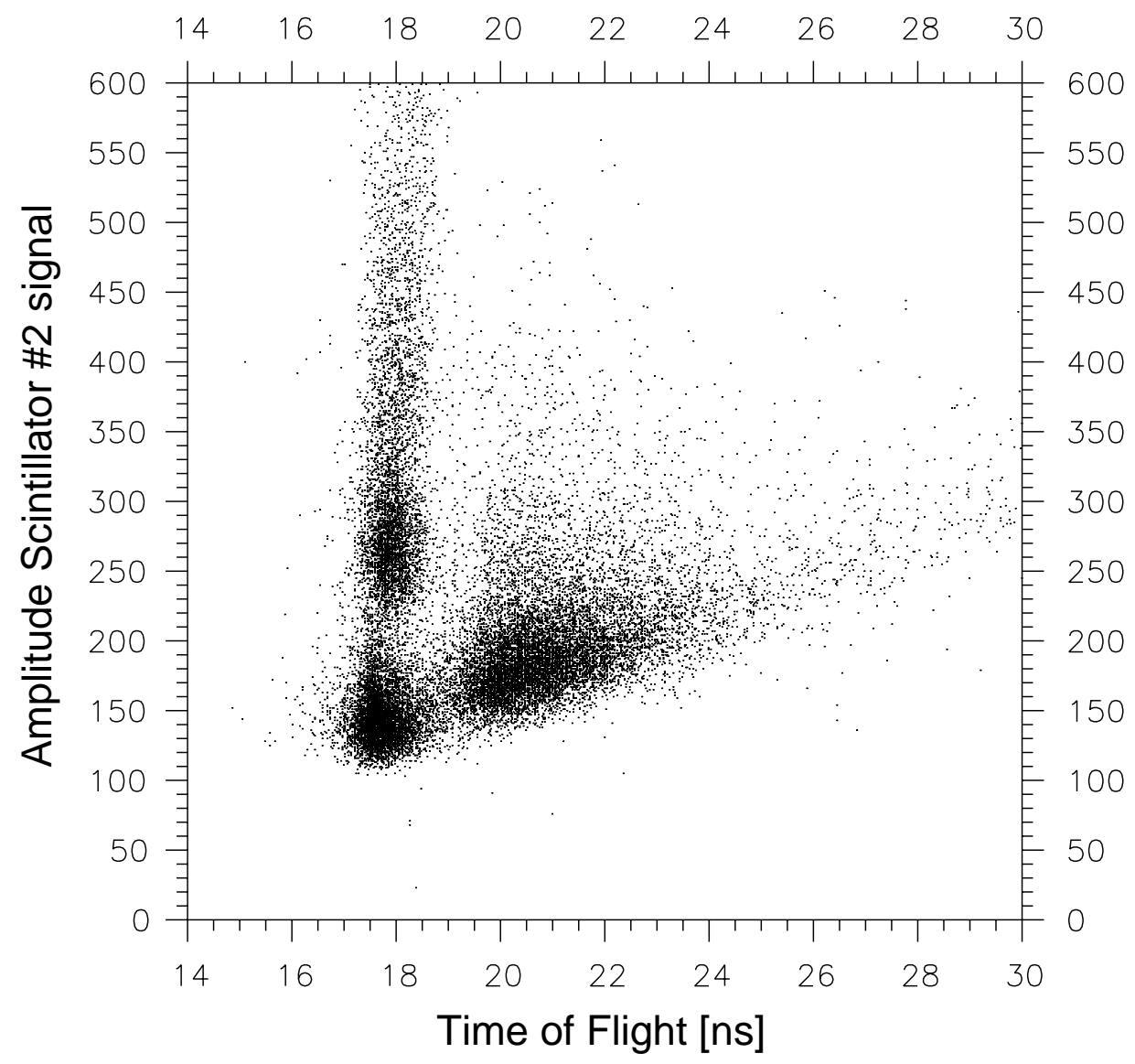

Figure 3: Two dimensional display of time-of-flight vs. scintillator signal amplitude 


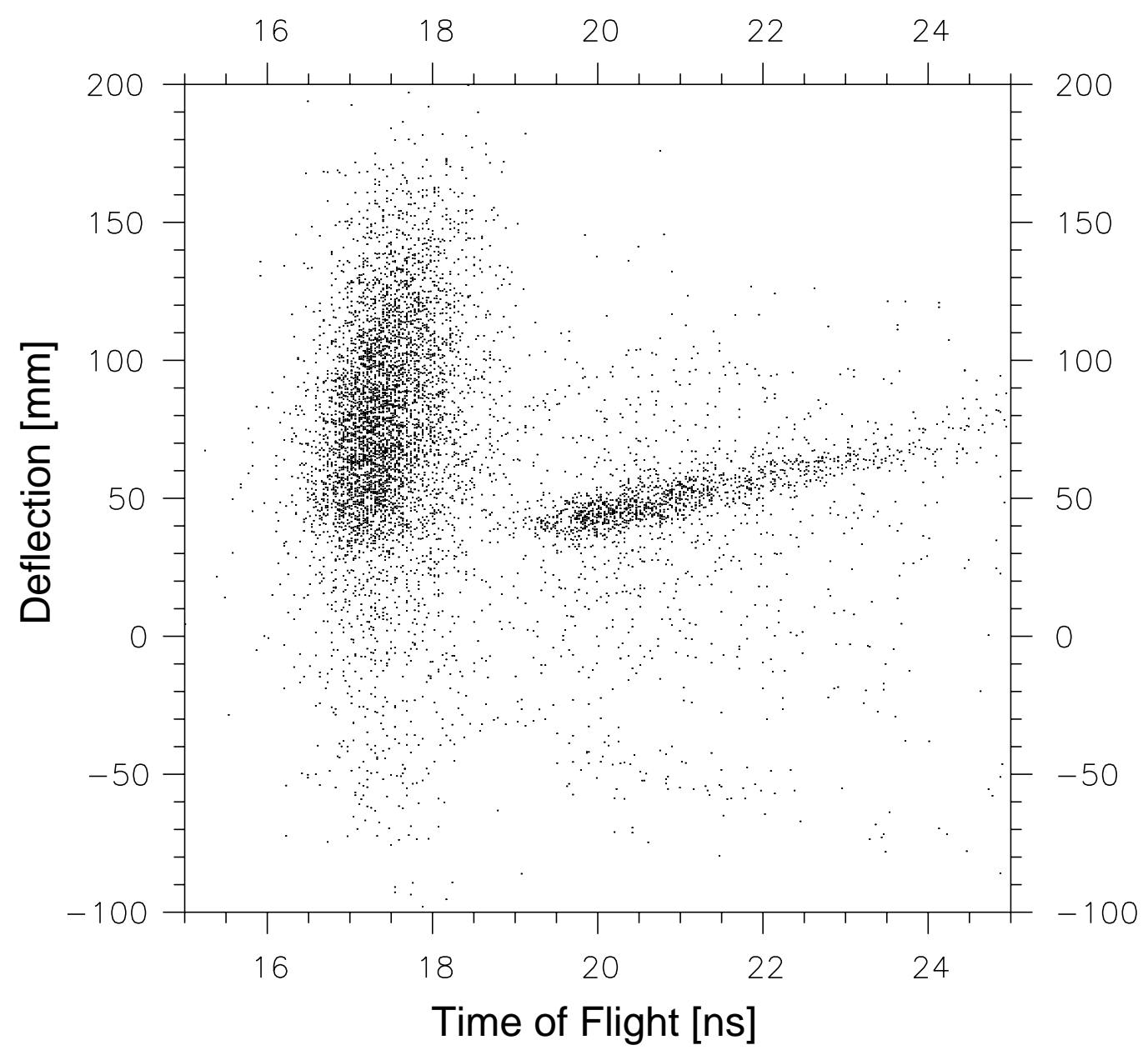

Figure 4: Two dimensional display of time-of-flight vs. deflection due to the magnetic dipole 


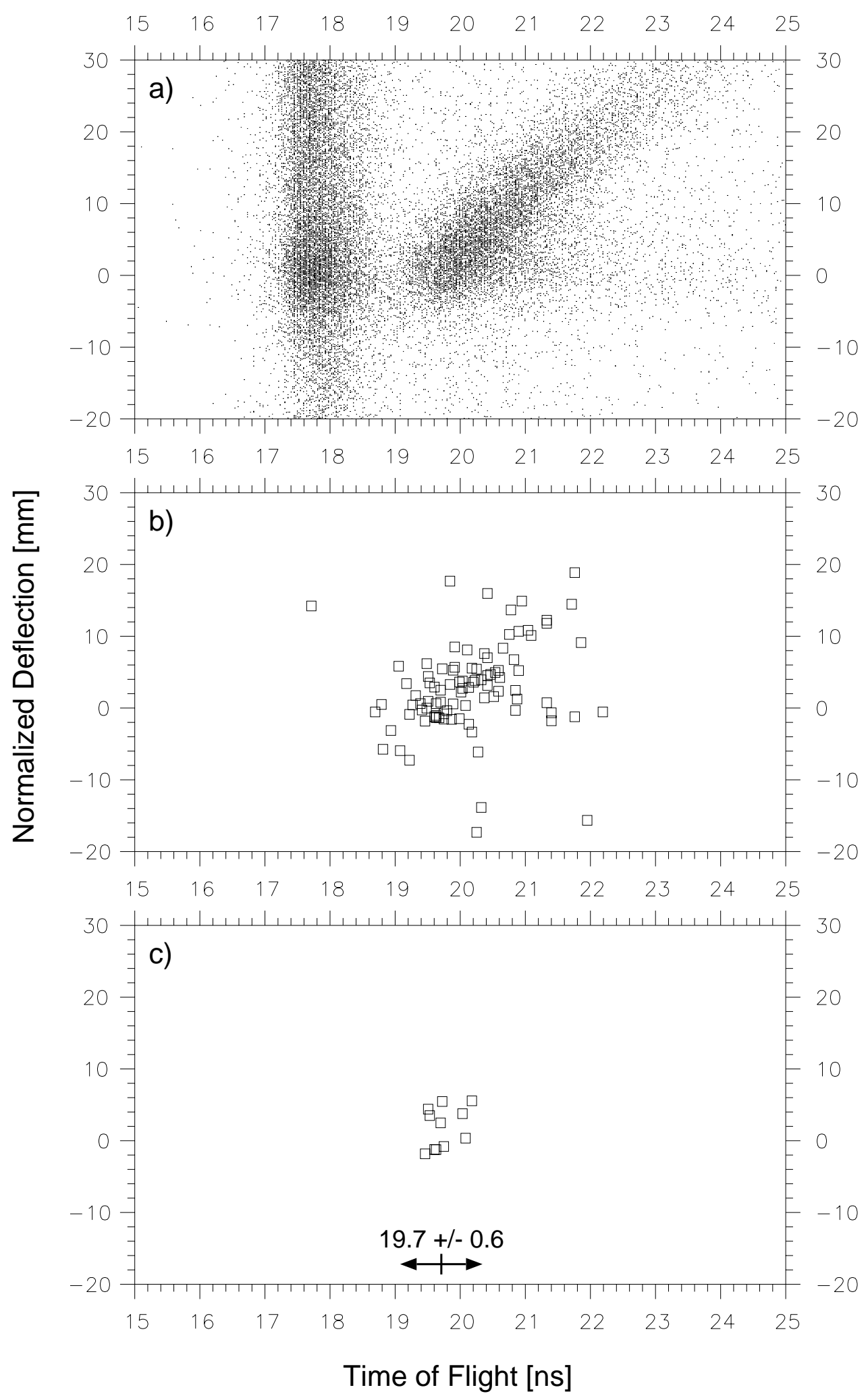

Figure 5: Time-of-flight vs. deflection spectra, steps of different cuts were applied to the raw data, see text for details 


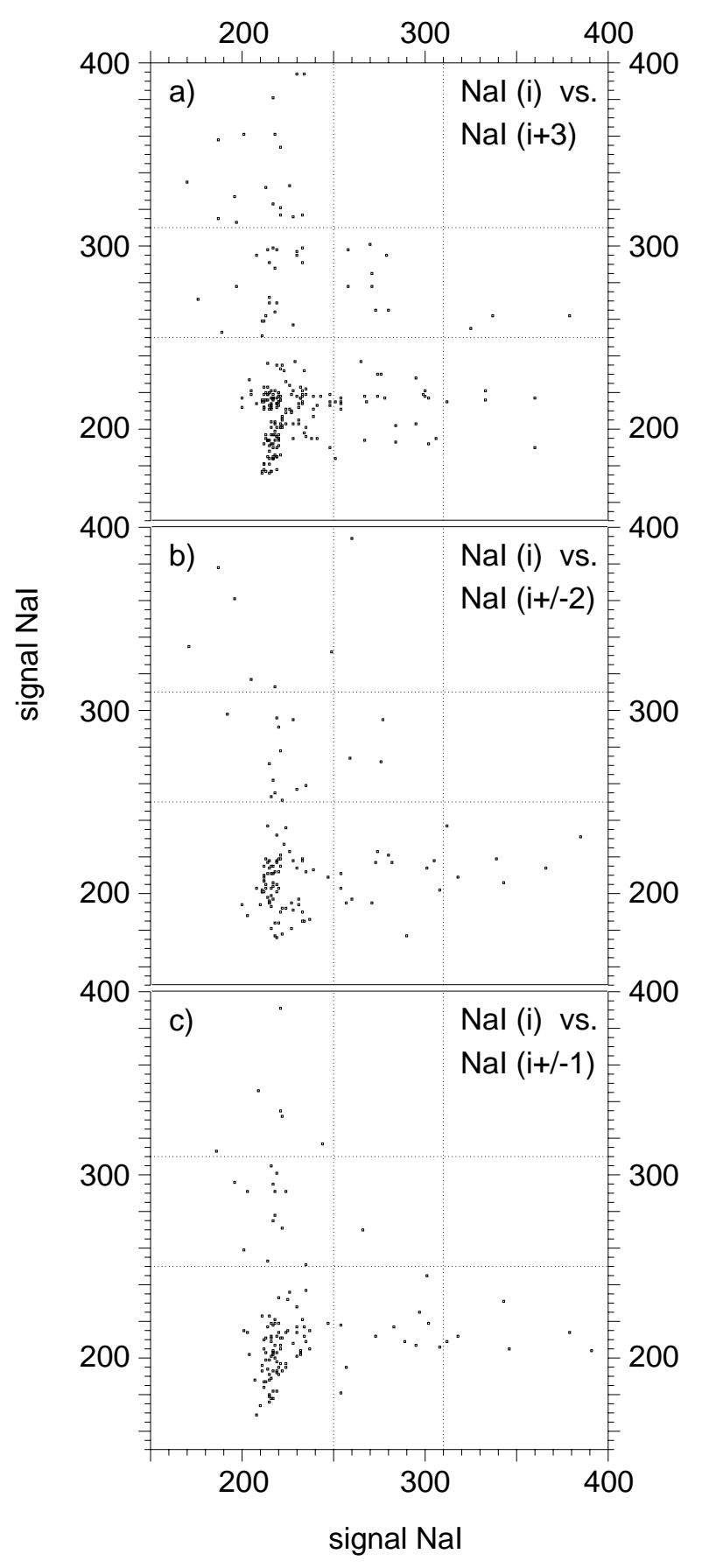

Figure 6: Two dimensional display of energy signals from

a) all opposite $\mathrm{NaI}$ crystals

b) non-adjacent and non-opposite NaI crystals

c) neighboring $\mathrm{NaI}$ crystals

of the six-fold segmented $\mathrm{NaI}$ detector. The energy cuts applied for the selection of $\mathrm{e}^{+}$annihilation are shown by dotted lines 


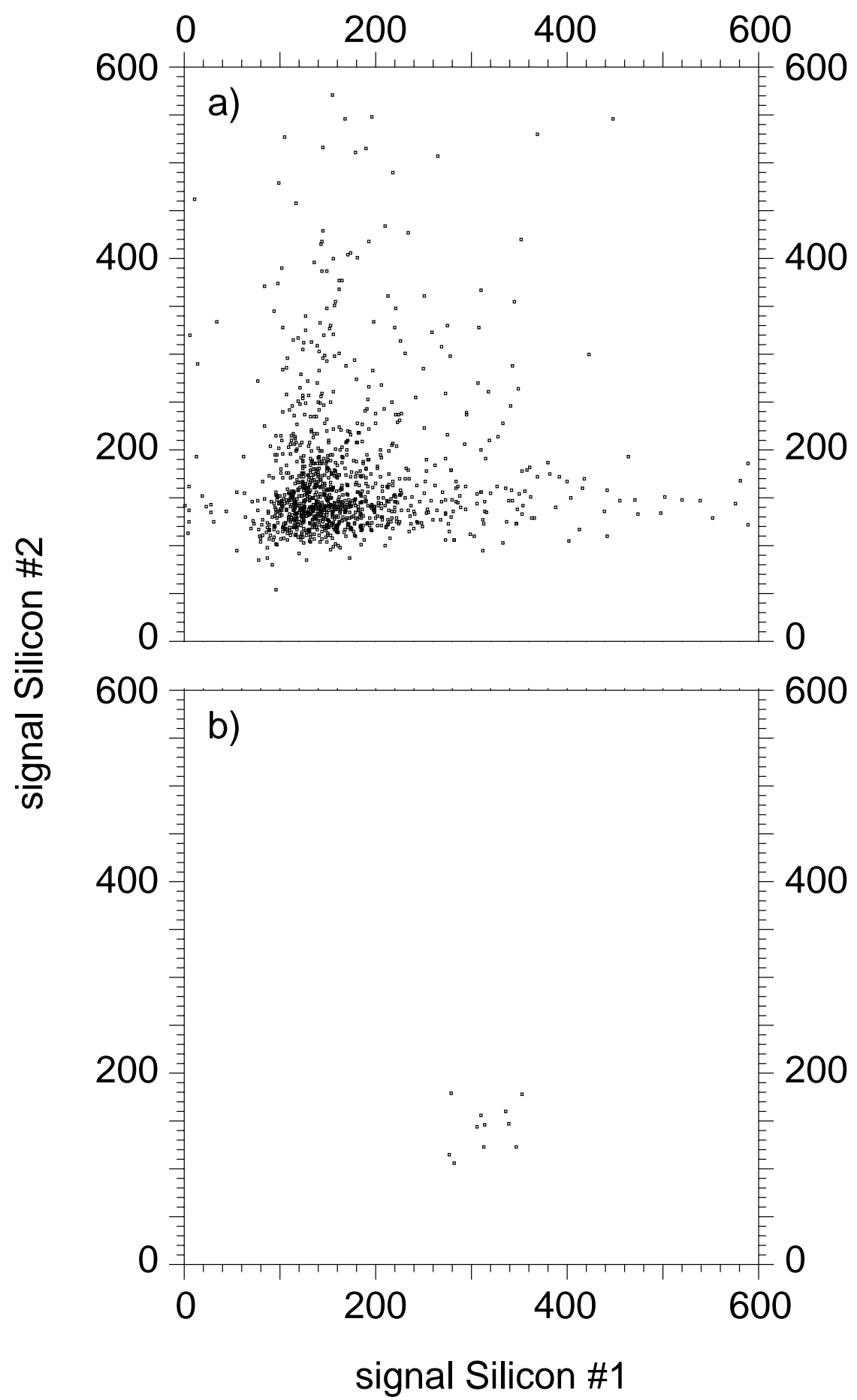

Figure 7: Signal amplitudes of the first versus the second silicon detector

a) sum over all $\overline{\mathrm{p}}$ 's identified by the magnetic spectrometer and the time-of-flight

b) events which are candidates for the production of $\overline{\mathrm{H}}^{0}$ 


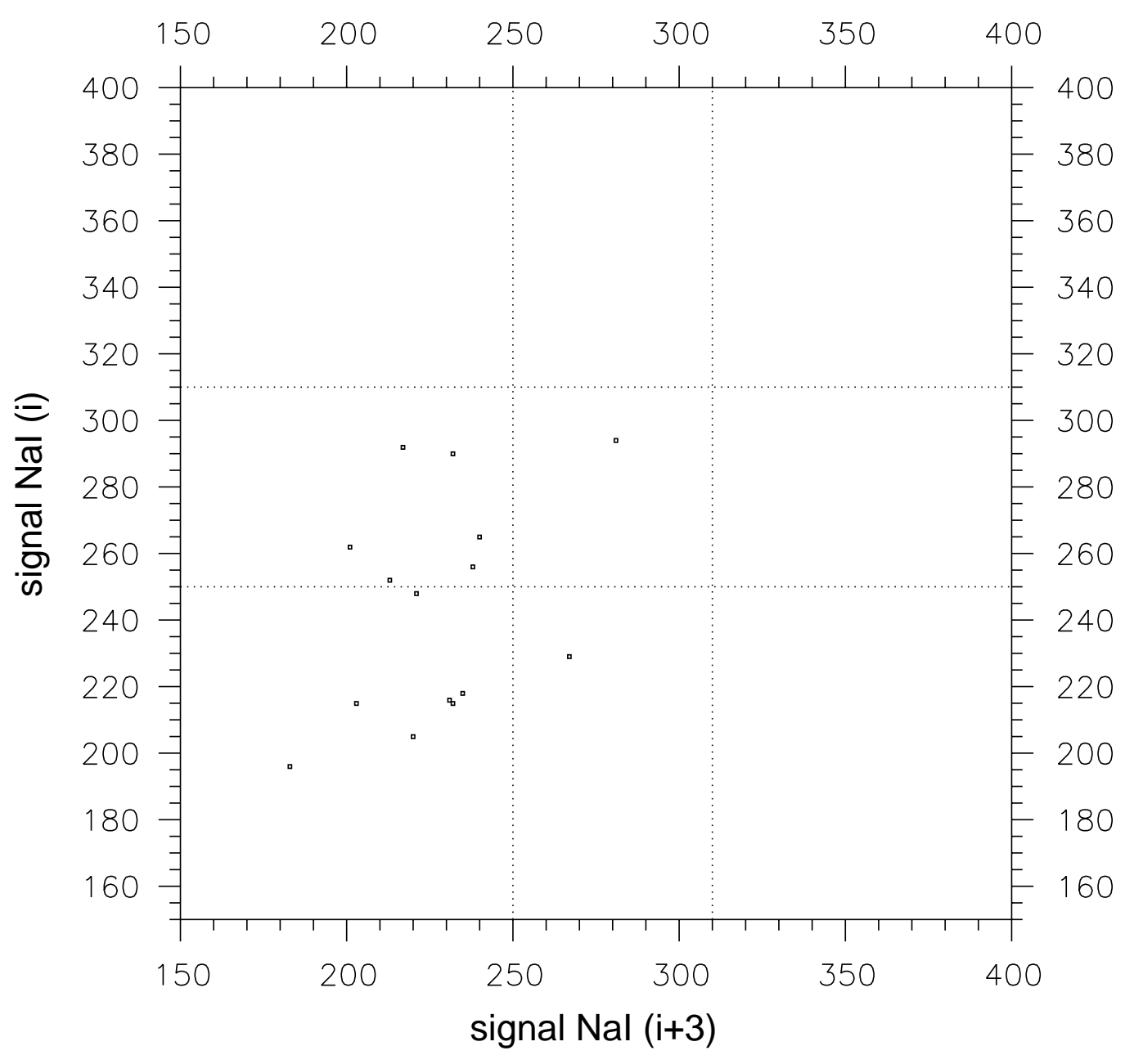

Figure 8: Two dimensional display of energy signals from all opposite $\mathrm{NaI}$ crystals in coincidence with no and any signal from the first two and the third silicon detector, respectively 


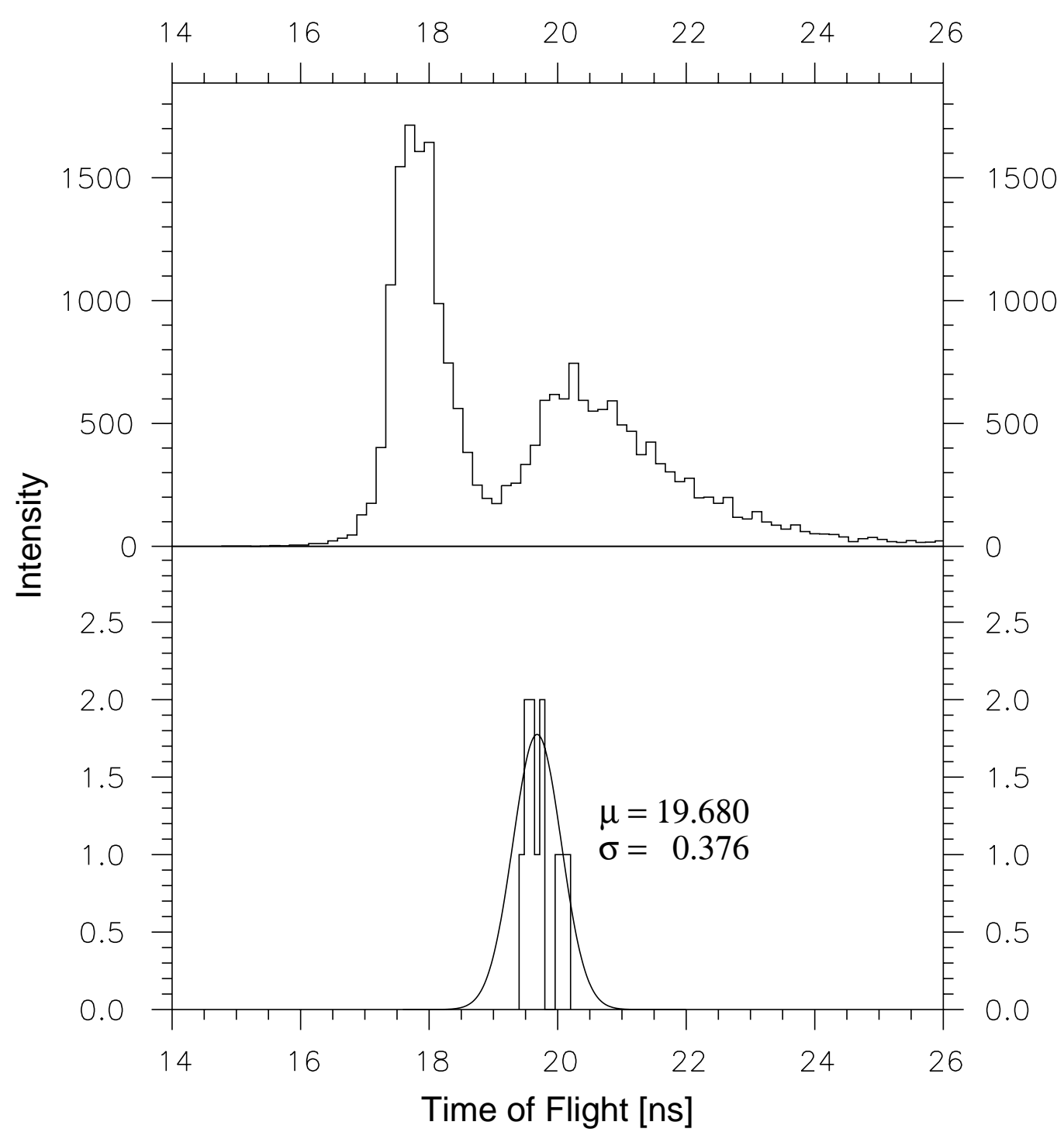

Figure 9: Comparison of the time-of-flight for all events to those extracted from the events with $\overline{\mathrm{H}}^{0}$ signature 